\title{
Measurement of the mass and width of the charmed meson $D^{*+}(2010)$
}

\author{
The ACCMOR Collaboration
}

Amsterdam-Bristol-CERN-Cracow-Munich-Rutherford-Valencia Collaboration

S.Barlag ${ }^{5 a}$, H.Becker ${ }^{5 b}$, A.Bożek ${ }^{4}, T$. Böhringer ${ }^{3 c}$, M.Bosman $^{5}$, V.Castillo ${ }^{3 d}$, V.Chabaud ${ }^{3}$, C.Damerell ${ }^{6}$, C.Daum ${ }^{1}$, H.Diet ${ }^{5}$, A.Gillman ${ }^{6}$, R.Gilmore ${ }^{2}$, T.Gooch ${ }^{2}$, L.Görlich ${ }^{4}$, P.Gras ${ }^{7}$, Z.Hajduk ${ }^{4}$, E.Higon ${ }^{7}$, D.P.Kelsey ${ }^{3 e}$, R.Klanner ${ }^{5 f}$, S.Kwan ${ }^{3 g}$, B.Lücking ${ }^{5}$, G.Lütjens ${ }^{5}$, G.Lutz ${ }^{5}$, J.Malos ${ }^{2}$, W.Männer ${ }^{5}$, E.Neugebauer ${ }^{5 h}$, H.Palka ${ }^{4}$, M.Pepé ${ }^{6 i}$, J.Richardson ${ }^{6 j}$, K.Rybicki ${ }^{4}$, H.J.Seebrunner ${ }^{3}$, U.Stierlin ${ }^{5}$, H.G.Tiecke ${ }^{1}$, G.Waltermann ${ }^{5}$, S.Watts ${ }^{8}$, P.Weilhammer ${ }^{3}$, F.Wickens ${ }^{6}$, L.W.Wiggers ${ }^{1}$, M.Witek ${ }^{4}$, and T.Zeludziewicz ${ }^{4 k}$

\begin{abstract}
Using a high-resolution silicon vertex detector we have observed a very clean signal of $127 \mathrm{D}^{*+}$. After a careful study of the experimental resolution of our apparatus we have measured $m\left(D^{*+}\right)-$ $m\left(D^{0}\right)=(145.39 \pm 0.06 \pm 0.03) \mathrm{MeV}$. We have also obtained a 90\% CL upper limit to $\Gamma\left(D^{*+}\right)$ of $131 \mathrm{keV}$.
\end{abstract}

Submitted for publication in Phys. Lett. B.

1) NIKHEF-H,Amsterdam,NL

2) Univ. of Bristol,UK

3) CERN,Geneva,CH

4) Inst.of Nucl.Physics,Cracow,Poland

5) Max-Planck-Ins. f. Physik,Munich,FRG

6) Rutherford Appleton Lab.,Chilton,Didcot,UK

7) IFIC,CSIC and Univ of Valencia,Valencia,Spain

8) Brunel Univ.,Uxbridge,Mddx,UK a) Now at KNMI, De Bilt, NL

b) Now at Gesamthochschule,Saarbrucken,FRG

c) Now at University of Lausanne,Lausanne,CH

d) Now at IFIC,CSIC and Univ of Valencia, Valencia,Spain.

e) Now at Rutherford Appleton Lab., Chilton,Didcot,UK

f) Now at DESY,Hamburg,FRG

g) Now at FNAL, Batavia, USA

h) Now at Universitat-GH Siegen,FRG

i) Now at CERN,Geneva,Switzerland

j) Now at Univ.of Geneva,Geneva,CH

k) Now at University of Melbourne, Melbourne, Australia 



\section{Introduction}

Although the $D^{*}(2010)$ meson was discovered fifteen years ago [1] its width has not yet been measured. The tightest upper limit of $1.1 \mathrm{MeV}(90 \%$ confidence level (CL)) was published by the HRS collaboration [2] studying the shape of the mass difference $\Delta m=m\left(D^{*+}\right)-m\left(D^{0}\right)$ (throughout the paper a particle symbol stands for particle and antiparticle). This estimate of $\Gamma\left(D^{*+}\right)$ was obtained by subtracting quadratically the apparatus resolution from the width of the observed $\Delta m$ distribution. We describe here a measurement of the $D^{*+}$ mass and width based on an analysis of data from the NA32 experiment, in which we have obtained a sample of $127 \mathrm{D}^{*+}$ with a background of 17 events. Their production properties have already been published [3].

\section{The experiment}

The second phase of the NA32 experiment was performed at the CERN-SPS using a negative $230 \mathrm{GeV} / \mathrm{c}$ beam (96\% pions and $4 \%$ kaons) and a $2.5 \mathrm{~mm} \mathrm{Cu}$ target. Charm decays were reconstructed with an improved silicon vertex detector and a large-acceptance spectrometer. The latter consisted of two magnets, 48 planes of drift chambers and three multicellular Cerenkov counters allowing $\pi, K, p$ identification in a wide momentum range. The vertex detector consisted of a beam telescope (seven microstrip planes) and a vertex telescope with two charge-coupled devices and eight microstrip planes. The overall precision of our vertex detector allowed a purely topological charm search which is restricted neither to a limited number of decay modes nor to any mass window. As a byproduct we have observed $65000 K^{0}$ and $25000 \Lambda^{0}$ decays, all seen in the vertex detector. The results described in this letter are based on the full sample of 17 million triggers.

\section{Data analysis, signal and background}

Event reconstruction is done in several steps (see ref.[4] for more details). First, all tracks are reconstructed in the drift chambers and particle identification is performed. Independently the beam track and the secondary tracks are reconstructed in the beam and vertex telescope, respectively. Then, tracks found in the drift chambers and in the vertex telescope are matched. Finally, the reconstruction of the primary vertex is performed. We only accept events with the primary vertex inside the target and at least two tracks not originating from the vertex. These tracks are then used as a seed to the search for one or more secondary vertices. The vertices should be between the target and the first microstrip plane ( $65 \mathrm{~mm}$ from the target). In addition we demand the vector sum of the momenta of all particles originating from the secondary vertex to pass through the primary one. This results in about 1200 fully reconstructed decays of $D^{0}, D^{+}, D_{s}^{+}$, $\Lambda_{c}^{+}, \Xi_{c}^{+}$and $\Xi_{c}^{0}$ (see refs[4, 5, 6] for details). In our study of $D^{*+} \rightarrow D^{0}\left(K^{-} \pi^{+}\right) \pi^{+}$or $D^{*+} \rightarrow D^{0}\left(K^{-} \pi^{+} \pi^{+} \pi^{-}\right) \pi^{+}$we demand: 
- a decay vertex including an identified $K^{-}$and one or three pions

- the effective mass of the $K^{-} \pi^{+}$or $K^{-} \pi^{+} \pi^{+} \pi^{-}$system to be within 2 sigma of the $D^{0}$ mass

- an additional pion $\pi_{e x t}^{+}$originating from the primary vertex and not identified as $K^{+}$or $p$

- $m\left(D^{0} \pi_{e x t}^{+}\right)<2015 \mathrm{MeV}$

Two events for which ambiguous $D^{0}$ vertices are found have been discarded. Contrary to the $e^{+} e^{-}$experiments we do not need kinematical cuts to clean our signal. Among our $127 D^{*+}$ events there are 26 with $D^{0} \rightarrow K^{-} \pi^{+}$and 101 with $D^{0} \rightarrow K^{-} \pi^{+} \pi^{+} \pi^{-}$. The $\Delta m$ distribution for these events is shown in Fig.1.

We estimate the background in the signal region by studying the effective mass distribution of a $D^{0}$ from one event combined with the $\pi_{e x t}^{+}$from another event. This "event mixing" method yields 17.2 background events in good agreement with 20 "wrong sign" $\left(D^{0} \pi_{e x t}^{-}\right)$events observed in the signal region in our experiment. Since the first estimate is statistically superior we use it in our analysis.

\section{Experimental resolution and acceptance correc- tions}

An exact knowledge of the behaviour of our apparatus is of utmost importance for a study of a very narrow width like that of the $D^{*+}$. We have performed an extensive investigation of our resolution for several decay channels of strange or charmed particles always demanding the decay to occur before the first microstrip plane as for the $D^{0}$. From our measurement errors and from multiple scattering errors throughout the set-up we have derived analytically the expected error $\sigma_{i}$ of the effective mass $m_{i}$ or of the effective mass difference $\Delta m_{i}$ for each event in all investigated decay channels. Then these calculations were "calibrated" by studying the experimental distribution of the reduced mass

$$
w_{i}=\frac{m_{i}-<m>}{\sigma_{i}}
$$

or of the reduced mass difference

$$
w_{i}=\frac{\Delta m_{i}-<\Delta m>}{\sigma_{i}}
$$

for each decay channel. If $\sigma_{i}$ contains properly all errors the distribution of $w_{i}$ should be Gaussian with a standard deviation of unity; larger width indicates underestimation of our $\sigma_{i}$. The results are listed in Table 1 (the last line refers to the mass difference of the reconstructed $\Xi^{-}$and $\Lambda^{0}$ ). While this table shows reasonable consistency of our error calculations, it does demonstrate the presence of residual errors not accounted for by our formulae. It should be pointed out that the average momentum of strange particles $(20 \mathrm{GeV} / \mathrm{c})$ is lower than for the charmed ones $(60 \mathrm{GeV} / \mathrm{c})$. For subsamples of strange 
particles having an average momentum of $50 \mathrm{GeV} / \mathrm{c}$, we get standard deviations of $w_{i}$ around 1.08 .

We used the following procedure to tune $\sigma_{i}(\Delta m)$ for each event. It turns out that $\sigma_{i}$ is dominated ( $98 \%$ on the average) by the errors on the direction and the momentum of $\pi_{e x t}^{+}$. Similarly for the $\Lambda^{0}$ of Table $1 \sigma_{i}$ is dominated (94\% on the average) by the errors from the decay pion. The contribution of $\pi_{e x t}^{+}$to $\sigma_{i}(\Delta m)$ consists of 2 comparable terms: scattering inside the target and measurement errors on the track direction and its momentum. We calculate the first term with the precise formula of Lynch and Dahl [7]. We adjust the calculated value of the second term by multiplying it by the value measured for the standard deviation of $w_{i}\left(\Lambda^{0}\right)$ from a sample of $\Lambda^{0}$ for which the decay pion has a similar range of momentum as for $\pi_{e x t}^{+}$from $D^{*+}$. We determined this empirical factor to be 1.03 with an uncertainty of \pm 0.02 . If we just took the average empirical factor from Table 1 then our $90 \%$ CL upper limit for $\Gamma\left(D^{*+}\right)$ would become even smaller.

The acceptance corrections (cf. ref.[3] for details) are calculated with the help of a Monte Carlo program generating events according to the reaction $\pi^{-} \mathrm{Cu} \rightarrow D^{*+} \bar{D} X$ where $\bar{D}$ is a $2: 1$ mixture of $\bar{D}^{0}$ and $D^{-}$while $X$ stands for other particles produced in the interaction. $D^{*+}$ decays into the channel under consideration and $\bar{D}$ according to branching fractions given in the PDG[8] tables. The parameters of the other particles $X$ are read from a sample of interaction-trigger events recorded during the experiment. Then the generated and real tracks are merged and the resulting signals are used to simulate our trigger and particle identification. The $D^{*+}$ acceptance is $4.5 \%$ for $D^{0} \rightarrow K^{-} \pi^{+}$and $6.0 \%$ for $D^{0} \rightarrow K^{-} \pi^{+} \pi^{+} \pi^{-}$. This acceptance does not depend on the $D^{*+}$ mass in the region under consideration thus enabling us to study the shape of $m\left(D^{0} \pi_{e x t}^{+}\right)$without any acceptance corrections.

\section{Results}

We use the maximum-likelihood method to determine the parameters of the $D^{*+}$. It is described by a $\mathrm{P}$-wave Breit-Wigner distribution with a mass-dependent width i.e. $\Gamma=\Gamma_{0}\left(q / q_{R}\right)^{3}$ where $q$ is the $\pi_{e x t}^{+}$momentum in the $D^{*+}$ rest system and $q_{R}$ is the corresponding value at the resonance. Usually one multiplies $\Gamma$ with $D\left(q_{R}\right) / D(q)$, where $D(q)=1+(q R)^{2}$ is the Blatt-Weisskopf function and $R=5 \mathrm{GeV}^{-1}$. However at such low momenta $\left(q_{R}=40 \mathrm{MeV} / \mathrm{c}\right)$ the Blatt-Weisskopf function is close to unity. On the other hand the third-power dependence is very important as it considerably raises the right-hand tail of the Breit-Wigner curve compared to the constant-width Breit-Wigner. The experimental resolution which appears to dominate the observed width of the $D^{*+}$ is described for each event by a Gaussian distribution with the calculated $\sigma_{i}$ corrected by the above-mentioned empirical factor of 1.03 . The maximum-likelihood fit yields $\Gamma_{0}$ consistent with zero, with a standard deviation (68\% CL) of $68 \mathrm{keV}$ and a $90 \%$ CL upper limit $^{1}$ of $131 \mathrm{keV}$ (cf. Fig 1).

\footnotetext{
${ }^{1}$ In order to find the $90 \%$ confidence level we fit the mass distribution with varying $D^{*+}$ mass and $\Gamma\left(D^{*+}\right)$ fixed to values from 0 to $300 \mathrm{keV}$ in steps of $5 \mathrm{keV}$. The values of the $\log (\mathrm{L})$ (L being the maximum-likelihood function) are then fitted to a second order polynomial in $\Gamma\left(D^{*+}\right)$. This polynomial defines a likelihood function, which is then integrated from zero to infinity and the $90 \%$ point is found on this curve.
} 
We have investigated the sensitivity of this upper limit to the values of the empirical factor and of the background estimate. Changing the empirical factors to 0.99 and 1.07 , we obtain $90 \%$ CL upper limits of $135 \mathrm{keV}$ and $129 \mathrm{keV}$ respectively. Keeping the empirical factor of 1.03 but changing the background by an estimated uncertainty of -3 and +3 events, we get upper limits of $139 \mathrm{keV}$ and $124 \mathrm{keV}$ respectively.

The fit also yields $\left\langle\Delta m>=<m\left(D^{*+}\right)-m(D 0)>=(145.39 \pm 0.06) \mathrm{MeV}\right.$ in good consistency with the PDG[8] value of $(145.44 \pm 0.06) \mathrm{MeV}$. We have studied the systematic error of $\Delta m$ coming from the uncertainty on the background and on the empirical factor as well as from other experimental effects. This error turns out to be dominated by the uncertainty on the currents of our two magnets. We estimated it to be $\pm 0.03 \mathrm{MeV}$.

We would like to comment here on the measurement of the width of $D^{* 0}$, the isospin partner of $D^{*+}$. The HRS collaboration published [2] an upper limit of $2.1 \mathrm{MeV}$ from the absence of a signal for the decay $D^{* 0} \rightarrow D^{+}\left(K^{-} \pi^{+} \pi^{+}\right) \pi_{e x t}^{-}$which could occur if the $D^{* 0}$ width was sufficiently large. In the mass region $m\left(D^{+} \pi_{e x t}^{-}\right)<2015 \mathrm{MeV}$ we observe two events while the expected background is 1.8. Assuming equal numbers of produced $D^{*+}$ and $D^{* 0}$ and following the HRS parametrization with a mass-independent width we would obtain $\Gamma\left(D^{* 0}\right)<0.5 \mathrm{MeV}(90 \% \mathrm{CL})$. In our opinion the proper parametrization for the decay $D^{*} \rightarrow D \pi$ is

$$
\Gamma\left(D^{*} \rightarrow D \pi\right)=C * g * q\left(D^{*} \rightarrow D \pi\right)^{3} / M\left(D^{*}\right)^{2}
$$

where:

$$
\begin{aligned}
D^{*} & =D^{*+}, D^{* 0} \\
D & =D^{0}, D^{+} \\
\pi & =\pi^{+}, \pi^{0} \\
C & =\text { Clebsch-Gordan coefficient }
\end{aligned}
$$

and the isospin invariance demands the same coupling constant $g$ for all charge combinations. Unfortunately using this formula (like we have done previously for the $D^{*+}$ ) we cannot give any upper limit for $\Gamma\left(D^{* 0}\right)$ since the minimum at $\Gamma\left(D^{* 0}\right)=0$ in the profile of $\log (\mathrm{L})$ is not deep enough. This however seems to be of lesser importance since the isospin invariance leads to $\Gamma\left(D^{* 0}\right)$ about $20 \%$ smaller than $\Gamma\left(D^{*+}\right)$ i.e. outside the range of the present experimental possibilities.

\section{Comparison with theoretical predictions}

We briefly review several predictions concerning the width of the $D^{*+}(2010)$. We will use the recent CLEO [9] results on the branching fractions rather than the PDG[8] values. Eichten et al.[10] calculated the partial width for the radiative decay $D^{*+} \rightarrow D^{+} \gamma$ to be $2.4 \mathrm{keV}$. This yields a total width of $(53 \pm 39) \mathrm{keV}$. Thews and Kamal[11] used SU(4) invariance and the experimental width of $\rho(770)$ and $K^{*}(890)$ to calculate the partial widths of pionic decays of $D^{*+}(2010)$. This leads to a total width of $(27 \pm 5) \mathrm{keV}$. The cloudy-bag-model calculation of Miller and Singer[12] yields $\Gamma\left(D^{*+}\right)=(82 \pm 5) \mathrm{keV}$. We 
see that our upper limit, though much smaller than the previous results, is still consistent with these predictions.

\section{Conclusions}

We have measured the total width of $D^{*+}(2010)$ to be less than $131 \mathrm{keV}(90 \% \mathrm{CL})$. Thus the $D^{*+}$ is by far the most narrow particle in the class of known strong decays. In addition we have measured the $D^{*+}-D^{0}$ mass difference to be $(145.39 \pm 0.06 \pm 0.03) \mathrm{MeV}$.

\section{Acknowledgements}

The authors are much indebted to Prof. Jan Kwiecinski for an enlightening discussion of the Breit-Wigner formula near threshold and to Prof. A.N.Kamal for a discussion of theoretical predictions concerning the width of $D^{*+}(2010)$.

\section{References}

[1] I.Peruzzi et al.,Phys. Rev. Lett., 37 (1976) 569.; G.J.Feldman et al.,Phys. Rev. Lett., 38 (1977) 1313.

[2] HRS collaboration, S.Abachi et al., Phys.Lett. B 212 (1988) 533.

[3] ACCMOR collaboration, S.Barlag et al., Z.Phys. C 49 (1991) 555.

[4] ACCMOR collaboration, S.Barlag et al., Z.Phys. C 48 (1990) 29.

[5] ACCMOR collaboration, S.Barlag et al., Phys.Lett. B 233 (1989) 522.

[6] ACCMOR collaboration, S.Barlag et al., Phys.Lett. B 236 (1990) 495.

[7] G.R.Lynch and O.I.Dahl, Nucl. Instr. and Meth. B58 (1991) 6.

[8] Particle Data Group: Review of Particle Properties, Phys.Lett. B 239 (1990).

[9] CLEO II collaboration, M.Procario et al., Physics in Collisions, Colmar, June 1991.

[10] E. Eichten et al., Phys. Rev. D21 (1980) 203.

[11] R.L.Thews and A.N.Kamal, Phys. Rev. D 32 (1985) 810.

[12] G.A.Miller and P.Singer, Phys. Rev. D 37 (1988) 2564. 
Table 1:

\begin{tabular}{l|c|c|c|}
\hline Decay channel & No. of events & Spread of $\sigma_{i}[\mathrm{MeV}]$ & Standard deviation of $w_{i}$ \\
\hline$D^{0} \rightarrow K^{-} \pi^{+}$ & 150 & 5 to 14 & $1.44 \pm 0.12$ \\
$D^{+} \rightarrow K^{-} \pi^{+} \pi^{+}$ & 250 & 4 to 13 & $1.14 \pm 0.07$ \\
$D^{0} \rightarrow K^{-} \pi^{+} \pi^{+} \pi^{-}$ & 445 & 4 to 11 & $1.17 \pm 0.05$ \\
$\Lambda_{c}^{+} \rightarrow p K^{-} \pi^{+}$ & 127 & 3 to 9 & $1.21 \pm 0.13$ \\
$\Lambda^{0} \rightarrow p \pi^{-}$ & 4500 & 0.6 to 1.6 & $1.01 \pm 0.01$ \\
$K_{s}^{0} \rightarrow \pi^{+} \pi^{-}$ & 4300 & 2 to 4 & $1.08 \pm 0.01$ \\
$\Xi^{-} \rightarrow\left(p \pi^{-}\right) \pi^{-}$ & 29 & 1 to 2.2 & $1.03 \pm 0.14$ \\
$\Xi^{-}-\Lambda^{0}$ & 29 & 0.75 to 2. & $1.30 \pm 0.17$
\end{tabular}

\section{Figure Captions}

Fig.1. Experimental distribution of $\Delta m=m\left(D^{*+}\right)-m\left(D^{0}\right)$ and result of maximumlikelihood fit. 


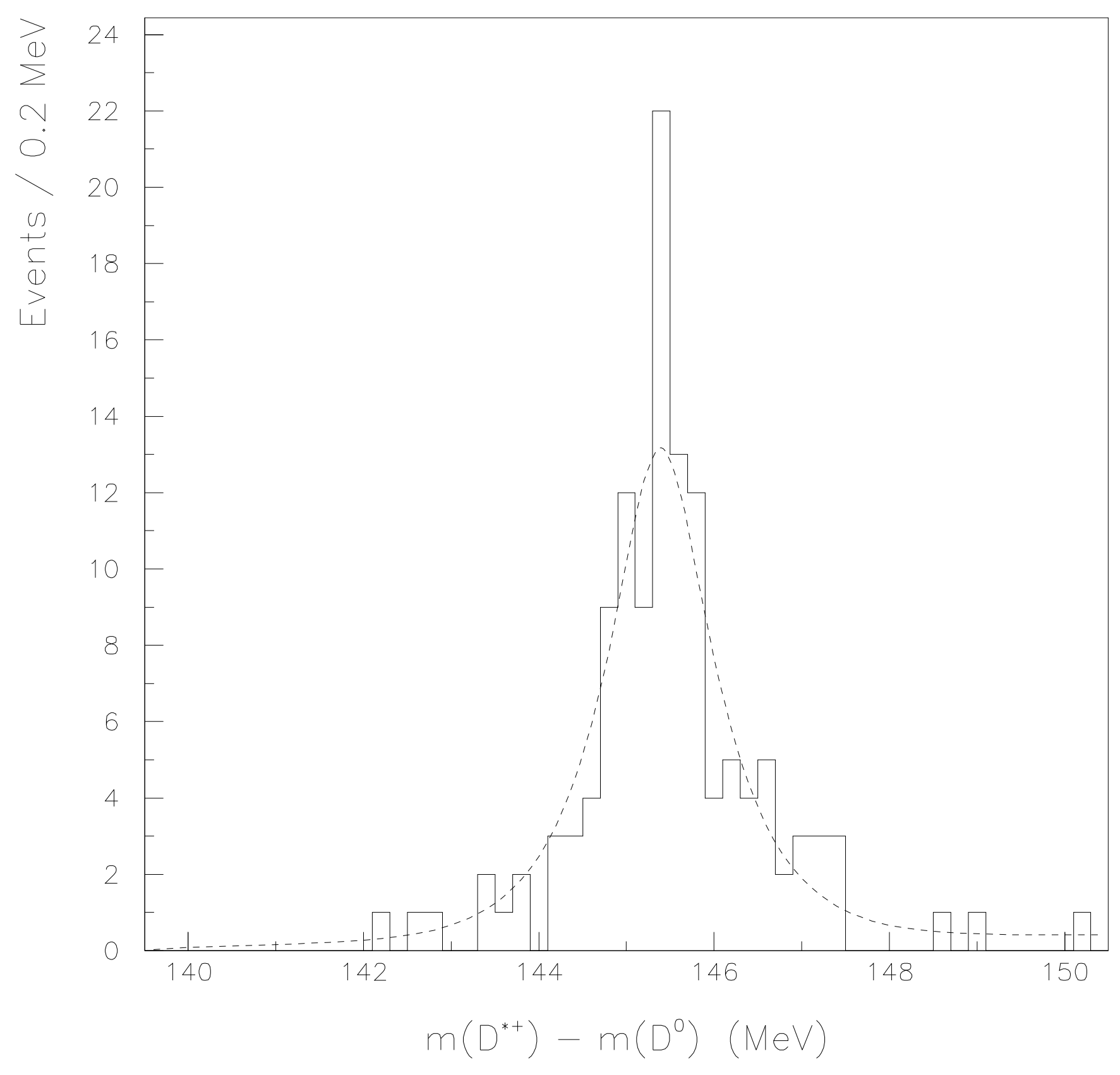

